\title{
Thermodynamic properties of xanthone: Heat capacities, phase-transition properties, and thermodynamic-consistency analyses using computational results*
}

\author{
Robert D. Chirico, ${ }^{a, \dagger}$ Andrei F. Kazakov, ${ }^{a}$ \\ ${ }^{a}$ Applied Chemicals and Materials Division \\ National Institute of Standards and Technology \\ Boulder, CO 80305-3337, U.S.A.
}

\footnotetext{
* Contribution of the National Institute of Standards and Technology, not subject to copyright in the United States.

${ }^{\dagger}$ Corresponding author. E-mail address: robert.chirico@nist.gov 


\begin{abstract}
Heat capacities and phase-transition properties for xanthone (IUPAC name $9 H$-xanthen9-one and Chemical Abstracts registry number [90-47-1]) are reported for the temperature range $5<T / \mathrm{K}<524$. Statistical calculations were performed and thermodynamic properties for the ideal gas were derived based on molecular geometry optimization and vibrational frequencies calculated at the B3LYP/6-31+G(d,p) level of theory. These results are combined with sublimation pressures from the literature to allow critical evaluation of inconsistent enthalpies of sublimation for xanthone, also reported in the literature. Literature values for the enthalpy of combustion of xanthone are reassessed, a revision is recommended for one result, and a new value for the enthalpy of formation of the ideal gas is derived. Comparisons with thermophysical properties reported in the literature are made for all other reported and derived properties, where possible.
\end{abstract}

Keywords: xanthone; computational chemistry; enthalpy of combustion, enthalpy of formation; enthalpy of fusion; enthalpy of sublimation; heat capacity; ideal-gas properties; phase-transition; triple point temperature 


\section{Introduction}

This article is part of a series in which we have reported studies of experimental and computed thermodynamic properties for aromatic ring systems, including an extensive series of azaaromatics (2-methylquinoline [1], 8-methylquinoline [1], 2,6dimethylquinoline [1], phenazine [2], acridine [2], phenanthridine [3], 1,10phenanthroline [3], and 7,8-benzoquinoline [3]), some hydroaromatics (1-phenyl and 2phenylnaphthalene [4]), and oxygen-containing compounds (9-fluorenone [5], and 1naphthol [6]). For this series, computations were performed at the B3LYP/6-31+G(d,p) model chemistry with excellent accord demonstrated between computed entropies for the ideal gas and those based on the experimental property measurements. In the present work, this approach is applied to xanthone (IUPAC name $9 H$-xanthen-9-one and Chemical Abstracts registry number [90-47-1]).

Results of our previous work [1-6] have demonstrated that for rigid fused-ring systems thermodynamic properties for the ideal-gas state can be computed with relative standard uncertainties near 0.1 percent. In the present work, we use this finding together with new condensed-phase thermodynamic properties determined with adiabatic calorimetry to assess and resolve inconsistencies between enthalpies of sublimation reported in the literature for xanthone [7-9]. These results allow evaluation of the enthalpy of formation of xanthone in the ideal gas state with high confidence, based on enthalpies of combustion reported in the literature $[7,8,10]$. As part of this assessment, an apparent error in one report [10] was detected and corrected.

Here, we report results of measurements of heat capacities and phase-transition properties for xanthone for the temperature range $5<T / \mathrm{K}<524$. The crystal-liquid-gas 
triple-point temperature and enthalpy of fusion were determined, together with properties associated with a small lambda-like transition in the solid state. Measured and derived thermodynamic property values are compared with those reported in the literature, where possible.

\section{Experimental}

\subsection{Materials}

The research group of Professor E. J. "Pete" Eisenbraun (retired) of Oklahoma State University purified the sample of xanthone used in this research. A commercial sample was recrystallized twice from ethanol (95 percent) and twice from a 1:1 by volume mixture of (hexane + ethyl acetate). The resulting crystals were filtered and dried by evacuation at room temperature. The mole fraction purity $x$ of the sample $(x=0.9999)$ was determined by fractional melting as part of the adiabatic calorimetric studies. Sample handling during purification and transfer was done under a dry atmosphere of argon, nitrogen, or helium.

\subsection{Physical constants and standards}

Property values for xanthone are reported in terms of molar mass $M=196.19 \mathrm{~g} \cdot \mathrm{mol}^{-1}$ [11] based on the formula $\mathrm{C}_{13} \mathrm{H}_{8} \mathrm{O}_{2}$ and the gas constant $R=8.3144621 \mathrm{~J} \cdot \mathrm{K}^{-1} \cdot \mathrm{mol}^{-1}$ adopted in 2010 by CODATA [12]. The platinum resistance thermometer used here was calibrated by comparison with a standard thermometer calibrated at the National Bureau of Standards (NBS), now the National Institute of Standards and Technology (NIST). Temperatures were measured in terms of IPTS-68 [13] and were converted to ITS-90 with published differences [14]. The thermometer was calibrated below $T / \mathrm{K}=13.81$ 
with the method of McCrackin and Chang [15]. Mass, time, electrical resistance, and potential difference were measured in terms of standards traceable to calibrations at NIST.

\subsection{Adiabatic calorimetry}

Heat-capacities, enthalpy increments, and the triple-point temperature for xanthone were measured with an adiabatic calorimetric system described previously [16]. Samplecontainer characteristics and sealing conditions are given in table 1. Energies were measured with a relative standard uncertainty of 0.01 percent. Temperatures were measured with a repeatability of $0.0001 \mathrm{~K}$, which represents the lower limit in the standard uncertainty for measured temperature increments. The standard uncertainty for a single temperature measurement was $0.005 \mathrm{~K}$, due to uncertainties associated with the thermometer calibration, and represents the lower limit for the standard uncertainty in reported absolute temperatures, including phase-transition temperatures. The energy increments to the filled platinum calorimeter were corrected for enthalpy changes in the empty calorimeter and for the helium exchange gas. Corrections for vaporization of the sample into the free space of the sealed vessel were estimated to be insignificant, so none were applied. The maximum correction to the measured energy for the helium exchange gas was 0.4 percent near $T / \mathrm{K}=5$. The approximate size of the correction for the enthalpy changes in the empty calorimeter is given in table 1 . 


\section{Experimental Results}

\subsection{Heat capacities, phase-transition properties, and derived thermodynamic functions for the condensed phases}

Measurements were made for the temperature range $(5<T / \mathrm{K}<524)$, and included determination of heat capacities for the solid and liquid phases, the triple-point temperature $T_{\mathrm{tp}}$, the molar enthalpy of fusion $\Delta_{\mathrm{cr}}^{\mathrm{l}} H_{\mathrm{m}}$, and the temperature of a lambdalike transition $T_{\text {tr }}$ in the solid state. The density of liquid xanthone under saturation pressure at temperature $T / \mathrm{K}=473\left\{\rho_{\text {sat }}=1090 \pm 20 \mathrm{~kg} \cdot \mathrm{m}^{-3}\right\}$ was determined from the sample mass and volume measured as part of the filling procedure for the calorimetric vessel.

Crystallization of xanthone in the calorimeter was initiated by slow cooling (approximately $7 \mathrm{mK} \cdot \mathrm{s}^{-1}$ ) the liquid sample to approximately $10 \mathrm{~K}$ below $T_{\mathrm{tp}}$. Complete crystallization was obtained by maintaining the sample under adiabatic conditions in the partially melted state (10 percent to 20 percent liquid) until ordering of the crystals was complete, as evidenced by the absence of spontaneous warming of the sample. In these experiments, no spontaneous warming was observed, indicating rapid and complete crystallization of the sample. The sample was then cooled at an effective rate of $7 \mathrm{mK} \cdot \mathrm{s}^{-1}$ to crystallize the remaining liquid and was thermally cycled from approximately $100 \mathrm{~K}$ to within $3 \mathrm{~K}$ of the triple-point temperature $\left(T_{\mathrm{tp}}=448.79_{5} \mathrm{~K}\right)$, where it was held for a minimum of $16 \mathrm{~h}$ to provide further tempering. All of the solid-phase measurements were performed upon crystals pre-treated in this way. Excellent repeatability (within 0.01 percent) was observed for three separate $\Delta_{\mathrm{cr}}{ }^{\mathrm{l}} H_{\mathrm{m}}$ determinations, which further indicates complete conversion to the crystalline state. 
The triple-point temperature $T_{\mathrm{tp}}$ and mole fraction purity $x$ for xanthone were determined by measurement of the equilibrium melting temperatures $T(F)$ as a function of fraction $F$ of the sample in the liquid state [17]. Equilibrium melting temperatures were determined by measuring temperatures for $\sim 1.5 \mathrm{~h}$ after an energy input and extrapolating to infinite time by assuming an exponential decay toward the equilibrium value. The observed temperatures at the end of the equilibration period were within $2 \mathrm{mK}$ of the calculated equilibrium temperatures for $F$ values listed in table 2 and used in the determination of $T_{\mathrm{tp}}$. Results showed no evidence for the presence of solid-soluble impurities. The calculated triple-point temperature and sample purity are included in table 2 .

The experimental heat capacities under vapor saturation pressure $C_{\text {sat,m }}$ determined by adiabatic calorimetry for xanthone are listed in table 3 and shown in figure 1 . The values listed in table 3 were corrected for pre-melting with the mole fraction impurity value given in table 2 . Relative expanded uncertainties ( 0.95 level of confidence) for the heat-capacity measurements $U_{\mathrm{r}}\left(C_{\mathrm{sat}, \mathrm{m}}\right)$ ranged from 0.03 at $\mathrm{T} / \mathrm{K}=5$, to 0.005 at $T / \mathrm{K}=$ 10 , and improved gradually to 0.001 for temperatures $T / \mathrm{K} \geq 30$. Extrapolation of the heat-capacity results to $T / \mathrm{K} \rightarrow 0$ was made with a plot of $C_{\mathrm{sat}, \mathrm{m}} / T$ against $T^{2}$ for temperatures below $T / \mathrm{K}=10$.

Equilibrium was reached in less than $1 \mathrm{~h}$ for all measurements in the liquid phase and for measurements in the solid phase below $T / \mathrm{K}=420$. As the triple-point temperature $\left(T_{\mathrm{tp}}=448.79_{5} \mathrm{~K}\right)$ was approached, equilibration periods increased gradually from $1 \mathrm{~h}$ near $T / \mathrm{K}=420$ to $6 \mathrm{~h}$ near $T / \mathrm{K}=445$. 
As seen in figure 1 , there is a small lambda-like transition near the temperature $T / \mathrm{K}$ $=304.5$. Equilibration times remained less than 1 hour throughout this region, and enthalpy measurements across the transition were highly reproducible, as shown in table 4. No hysteresis was observed. Measurements were made with 3-K temperature increments (Series 8) and the transition temperature $T_{\text {tr }}$ was derived through extrapolation of the heat capacities for each phase, followed by adjustment of the transition temperature to yield zero excess enthalpy for measurements made across the transition region with large $(\sim 20 \mathrm{~K})$ temperature increments (Series 9 and 11). The transition temperature so determined was $T_{\mathrm{tr}} / \mathrm{K}=304.5$ with an estimated expanded uncertainty of $0.5 \mathrm{~K}$ ( 0.95 level of confidence). No attempt was made to determine the detailed shape of the heat capacity curve in the transition region with temperature increments less than $\sim 3 \mathrm{~K}$, but as the variation of the heat capacity in the upper and lower phases was nearly linear with temperature, the assignment of the $T_{\text {tr }}$ value could be made with high certainty.

Experimental molar enthalpy measurements are summarized in table 4, which includes results used in the determination of the enthalpy of fusion, determination of $T_{\mathrm{tr}}$, and measurements in single-phase regions, which serve as consistency checks for the integrated heat-capacity values. Corrections for pre-melting [18] caused by impurities were made in these evaluations with the impurity $x_{\text {pre }}$ listed in table 1 . Results with the same series number $N$ in tables 3 and 4 were taken without interruption of adiabatic conditions.

Entropies and enthalpies under vapor saturation pressure for the condensed phases relative to that of the crystals at $T \rightarrow 0 \mathrm{~K}$ for xanthone are listed in table 5 . These were 
derived by integration of the smoothed heat capacities corrected for pre-melting, together with the entropy and enthalpy of fusion. All pre-melting corrections were made using standard methods [17].

\section{Discussion}

\subsection{Comparisons with literature melting temperatures and enthalpies of fusion}

The normal melting temperature $T_{\mathrm{m}}$ and enthalpy of fusion $\Delta_{\mathrm{cr}}^{\mathrm{l}} H_{\mathrm{m}}$ for xanthone were reported by Sabbah and El Watik [7] $\left\{T_{\mathrm{m}} / \mathrm{K}=(449.63 \pm 0.3), \Delta_{\mathrm{cr}}{ }^{\mathrm{l}} H_{\mathrm{m}} /\left(\mathrm{kJ} \cdot \mathrm{mol}^{-1}\right)=(26.12\right.$ $\pm 0.24)\}$ and Monte et al. $\left\{T_{\mathrm{m}} / \mathrm{K}=(448.3 \pm 0.1), \Delta_{\mathrm{cr}}{ }^{\mathrm{l}} H_{\mathrm{m}} /\left(\mathrm{kJ} \cdot \mathrm{mol}^{-1}\right)=(26.6 \pm 0.2)\right\}$ using differential calorimetric methods. The melting temperatures are within $0.8 \mathrm{~K}$ of the triple-point temperature $T_{\text {tp }}$ determined here (table 2). For the enthalpies of fusion, the value reported by Monte et al.[9] is 3 percent low and that reported by Sabbah and El Watik [7] is 5 percent low. Such differences are common in comparisons of results from differential calorimetric methods with those of high-precision adiabatic calorimetry. It is also common that uncertainties reported with the differential methods are underestimated. There are numerous reports of $T_{\mathrm{m}}$ for xanthone in the literature, and these are, generally, within a few Kelvins of the value of $T_{\mathrm{tp}}$ reported here. Such differences are expected due to the relatively large uncertainties associated with the methods used for the literature values. In addition, the quality of the sample used in the present research far exceeds that used in most literature reports of normal melting temperature. 


\subsection{Assessment of literature enthalpies of sublimation through analysis of thermodynamic consistency}

As noted in the introduction, we have demonstrated previously [1-6] that thermodynamic properties in the ideal-gas state for rigid fused-ring systems, such as xanthone, can be computed with relative uncertainties near 0.1 percent. Recently, this was demonstrated recently for the 3-ring ketone, 9-fluorenone [5]. Vibrational frequencies for xanthone were calculated at the B3LYP/6-31+G(d,p) model chemistry with the Gaussian 09 software [19]. We have demonstrated for many compounds [1-6] that the single scale factor $F=0.975$ for the vibrational frequencies provides excellent agreement (within 0.1 percent) between computed ideal-gas entropies and those derived from calorimetric and thermophysical-property measurements. We also provided further justification for this scale factor through comparisons of computed and experimental gas-phase vibrational spectra [2] for quinoline and isoquinoline reported in the literature for all frequencies $\bar{v}$ less than $2000 \mathrm{~cm}^{-1}$. Higher frequencies, which are all greater than $3000 \mathrm{~cm}^{-1}$ for most molecules, were not considered important in the comparison because they make no significant contribution to thermodynamic properties in the temperature range of interest in this research. The scale factor $F=0.975$ and symmetry number 2 were used for xanthone, and the calculated vibrational frequencies and rotational constants are given in table 6 . There is general agreement between the calculated values and those measured experimentally for condensed phases by Zwarich and Binbrek [20], Sinha et al.[21], and Connors and Fratini [22]. There are no gas-phase spectra available for xanthone to allow direct comparison with the vibrational frequencies computed here. 
The enthalpy of sublimation for xanthone at given temperature $T$ can be calculated with the relationship

$$
\Delta_{\mathrm{cr}}^{\mathrm{g}} H_{\mathrm{m}}=T\left\{\Delta_{0}^{T} S_{\mathrm{m}}^{\mathrm{o}}(\text { ideal })-\Delta_{0}^{T} S_{\mathrm{m}}\{\mathrm{cr}(\mathrm{I})\}-S_{\mathrm{comp}}\right\}
$$

where $\Delta_{0}^{T} S_{\mathrm{m}}^{\mathrm{o}}$ (ideal) is the standard entropy of the ideal gas computed with parameters given in table $6\left(p^{\circ}=101.325 \mathrm{kPa}\right)$ and the methods described in the text, $\Delta_{0}^{T} S_{\mathrm{m}}(\mathrm{cr})$ is the standard entropy of crystalline xanthone interpolated from table 5, and $S_{\text {comp }}$ is the entropy of compression $\ln \left(p / p^{\circ}\right)$, calculated with sublimation pressures reported by Monte et al.[9].

Sabbah and El Watik [7] reported a value of $\Delta_{\mathrm{cr}}^{\mathrm{g}} H_{\mathrm{m}}$ at $T / \mathrm{K}=353$ based on a direct calorimetric method, and Monte et al.[9] reported this property at $T / \mathrm{K}=352.22$, based on the slope of sublimation pressures measured with Knudsen effusion. The sublimation enthalpies do not vary rapidly with temperature, so these values may be compared directly with the value at $T / \mathrm{K}=352.2$ derived in this research with equation (1). The experimental and derived values are listed in table 7 . The value of $\Delta_{\mathrm{cr}}^{\mathrm{g}} H_{\mathrm{m}}$ reported by Monte et al.[9] is $1.8 \mathrm{~kJ} \cdot \mathrm{mol}^{-1}$ higher than that derived here; however, the uncertainty claimed for their value $\left(0.6 \mathrm{~kJ} \cdot \mathrm{mol}^{-1}\right.$ or $\sim 0.6$ percent $)$ is probably too small, based on expected uncertainties of at least 1 percent for the method used. Consequently, we conclude that the agreement is good. In contrast, the value reported by Sabbah and El Watik [7] is $5 \mathrm{~kJ} \cdot \mathrm{mol}^{-1}$ lower than that derived here. This value is either in error or the uncertainty reported by Sabbah and El Watik [7] is more than one order of magnitude too small.

A value for $\Delta_{\mathrm{cr}}^{\mathrm{g}} H_{\mathrm{m}}$ at $T / \mathrm{K}=298.15$ was reported by Freitas et al. [8] based on measurement of enthalpy differences between the gas at temperature $T / \mathrm{K}=417$ and 
crystals at $T / \mathrm{K}=298.15$ with Calvet microcalorimetry, combined with a computed enthalpy for the gas phase for the interval $T / \mathrm{K}=298.15$ to $T / \mathrm{K}=417$. The value reported by Freitas et al.[8] is in good agreement with that of this research, as seen in Table 7. The origin of the uncertainty given by Freitas et al. $\left(2.3 \mathrm{~kJ} \cdot \mathrm{mol}^{-1}\right)$ is not clear, because the reported uncertainty for their measured enthalpy difference is $1.3 \mathrm{~kJ} \cdot \mathrm{mol}^{-1}$. Additional uncertainty may have been added due to unknown reliability of the computational methods used. The computational methods used in the present research have been validated [1-6] and shown to produce gas-phase properties with small uncertainties, so we revised the value of $\Delta_{\mathrm{cr}}^{\mathrm{g}} H_{\mathrm{m}}$ at $T / \mathrm{K}=298.15$ reported by Freitas $e t$ $a l .[8]$ by substituting the computational results of the present research and decreasing the uncertainty. The revised value $(104.2 \pm 1.3) \mathrm{kJ} \cdot \mathrm{mol}^{-1}$ is in excellent agreement with that of the present research $(103.26 \pm 0.4) \mathrm{kJ} \cdot \mathrm{mol}^{-1}$, justifying the revised smaller uncertainty.

\subsection{Assessment of literature enthalpies of combustion}

The enthalpy of combustion for xanthone has been studied with oxygen-bomb calorimetry by Freitas et al.[8], Sabbah and El Watik [7], and Kim et al.[10]. The values reported by Freitas et al.and Sabbah and El Watik are in excellent agreement, while the value reported by $\mathrm{Kim}[10]$ is $5 \mathrm{~kJ} \cdot \mathrm{mol}^{-1}$ higher. The value reported by Kim et al.[10] includes a significant "impurity correction" that we believe was added in error. The sample used by Kim et al.[10] was carefully purified by crystallization from ethanol and dried, as was the sample in the present research. Kim et al.also used a gas chromatograph/mass spectrometer to look for organic impurities, and none were found. In addition, no mention of an impurity correction is given in the text of the article. We 
recalculated the enthalpy of formation of xanthone with the experimental results reported by Kim et al.[10], but without the "impurity correction," and the revised value is given at the bottom of table 8 . Agreement of the revised value with those of Freitas et al.[8] and Sabbah and El Watik [7] is excellent; within $0.6 \mathrm{~kJ} \cdot \mathrm{mol}^{-1}$, in contrast to a difference of $5 \mathrm{~kJ} \cdot \mathrm{mol}^{-1}$ with the "correction" included.

\subsection{Enthalpy of formation of xanthone in the ideal-gas state}

The enthalpy of formation of xanthone in the ideal-gas state is now calculated with the weighted average of the enthalpies of formation for the crystals reported by Freitas et al.[8], Sabbah and El Watik [7], and Kim et al.[10] (revised) $\left\{\Delta_{\mathrm{f}} H_{\mathrm{m}}^{\mathrm{o}}(\mathrm{cr})=-(197.0 \pm\right.$ $4.0)\}$, together with the enthalpy of sublimation derived in this research $\left\{\Delta_{\mathrm{cr}}^{\mathrm{g}} H_{\mathrm{m}}=\right.$ $(103.6 \pm 0.4) \mathrm{kJ}^{\mathrm{mol}}{ }^{-1}($ table 7$\left.)\right\}$ to yield $\Delta_{\mathrm{f}} H_{\mathrm{m}}^{\mathrm{o}}($ ideal gas $\left.)=-(93.4 \pm 4.0)\right\}$

\section{Conclusion}

Computational chemistry is a powerful tool for evaluation of thermodynamic properties in the ideal-gas state, particularly for rigid molecules, such as xanthone. In this research we have demonstrated that computed gas-phase properties with validated uncertainties can provide key information that cannot be obtained from other sources for thermodynamic-consistency analysis and literature assessments.

\section{Acknowledgements}

We acknowledge the contributions of I. Alex Hossenlopp in vapor-transfer of the sample prior to heat-capacity studies and Stephen E. Knipmeyer for maintenance of all apparatus. The authors gratefully acknowledge the Office of Fossil Energy of the U.S. Department of Energy (DOE) for financial support of the experimental studies. This 
research was funded within the Processing and Downstream Operations section of the Advanced Oil Recovery (AOR) program. The experiments were completed through BDM-Oklahoma under its contract with DOE for Management and Operations of the National Oil and Related Programs (NORP), Contract Number DE-AC22-94C91008. Manuscript preparation and all computations were done at NIST. 


\section{References}

[1] R.D. Chirico, R.D. Johnson III, W.V. Steele, J. Chem. Thermodyn. 39 (2007) 698711.

[2] R.D. Chirico, A.F. Kazakov, W.V. Steele, J. Chem. Thermodyn. 42 (2010) 571581.

[3] R.D. Chirico, A.F. Kazakov, W.V. Steele, J. Chem. Thermodyn. 42 (2010) 581590.

[4] R.D. Chirico, A.F. Kazakov, W.V. Steele, J. Chem. Thermodyn. 73 (2014) 241254.

[5] R.D. Chirico, A.F. Kazakov, W.V. Steele, J. Chem. Thermodyn. 54 (2012) 278287.

[6] R.D. Chirico, W.V. Steele, A.F. Kazakov, submitted to J. Chem. Thermodyn. (2014).

[7] R. Sabbah, L. El Watik, Bull. Soc. Chim. Fr. 4 (1988) 626-630.

[8] V.L.S. Freitas, J.R.B. Gomes, M.D.M.C. Ribeiro da Silva, J. Therm. Anal. Calorim. 97 (2009) 827-833.

[9] M.J.S. Monte, C.A.D. Sousa, J.M.S. Fonseca, L.M.N.B.F. Santos, J. Chem. Eng. Data 55 (2010) 5264-5270.

[10] K.Y. Kim, R.E. Winans, W.N. Hubbard, C.E. Johnson, J. Phys. Chem. 82 (1978) 402-405.

[11] M.E. Wieser, N. Holden, T.B. Coplen, J.K. Böhlke, M. Berglund, W.A. Brand, P. De Bièvre, M. Gröning, R.D. Loss, J. Meija, T. Hirata, T. Prohaska, R. Schoenberg, G. O'Connor, T. Walczyk, S. Yoneda, X-K. Zhu, Pure Appl. Chem. 85 (2013) 1047-1078.

[12] 2010 CODATA Recommended Values: http://physics.nist.gov/cgi-bin/cuu/Value?r (Accessed March 8, 2012).

[13] Metrologia 5 (1969) 35-44. 
[14] R.N. Goldberg, R.D. Weir, Pure Appl. Chem. 64 (1992) 1545-1562.

[15] F.L. McCrackin, S.S. Chang, Rev. Sci. Instrum. 46 (1975) 550-553.

[16] W.V. Steele, D.G. Archer, R.D. Chirico, W.B. Collier, I.A. Hossenlopp, A. Nguyen, N.K. Smith, B.E. Gammon, J. Chem. Thermodyn. 20 (1988) 1233-1264

[17] J.P. McCullough, G. Waddington, Anal. Chim. Acta 17 (1957) 80-96.

[18] E.F. Westrum Jr., G.T. Furukawa, J.P. McCullough, in: J.P. McCullough, D.W. Scott (Eds.), Experimental Thermodynamics, vol. 1, Butterworths, London, 1968 (Chapter 5).

[19] M. J. Frisch, G. W. Trucks, H. B. Schlegel, G. E. Scuseria, M. A. Robb, J. R. Cheeseman, G. Scalmani, V. Barone, B. Mennucci, G. A. Petersson, H. Nakatsuji, M. Caricato, X. Li, H. P. Hratchian, A. F. Izmaylov, J. Bloino, G. Zheng, J. L. Sonnenberg, M. Hada, M. Ehara, K. Toyota, R. Fukuda, J. Hasegawa, M. Ishida, T. Nakajima, Y. Honda, O. Kitao, H. Nakai, T. Vreven, J. A. Montgomery, Jr., J. E. Peralta, F. Ogliaro, M. Bearpark, J. J. Heyd, E. Brothers, K. N. Kudin, V. N. Staroverov, T. Keith, R. Kobayashi, J. Normand, K. Raghavachari, A. Rendell, J. C. Burant, S. S. Iyengar, J. Tomasi, M. Cossi, N. Rega, J. M. Millam, M. Klene, J. E. Knox, J. B. Cross, V. Bakken, C. Adamo, J. Jaramillo, R. Gomperts, R. E. Stratmann, O. Yazyev, A. J. Austin, R. Cammi, C. Pomelli, J. W. Ochterski, R. L. Martin, K. Morokuma, V. G. Zakrzewski, G. A. Voth, P. Salvador, J. J. Dannenberg, S. Dapprich, A. D. Daniels, O. Farkas, J. B. Foresman, J. V. Ortiz, J. Cioslowski, and D. J. Fox, Gaussian 09, Revision C.01, Gaussian, Inc., Wallingford CT, 2010.

[20] R. Zwarich, O.S. Binbrek, Spectrochimica Acta A, 41 (1985) 537-544.

[21] H.K. Sinha, L. Chantranupong, R.P. Steer, J. Mol. Spectrosc. 169 (1995) 302-314. [22] R.E. Connors, C.M. Fratini, J. Mol. Struct. 553 (2000) 235-241. 
TABLE 1

Calorimeter and sample characteristics for adiabatic calorimetric studies on xanthone ${ }^{a}$

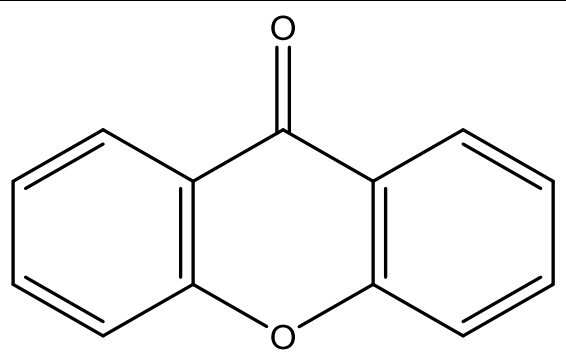

$m / \mathrm{g}$

47.893

$V_{\mathrm{i}}(298.15 \mathrm{~K}) / \mathrm{cm}^{3}$

61.78

$T_{\text {cal }} / \mathrm{K}$

298.2

$p_{\text {cal }} / \mathrm{kPa}$

4.53

$r\left(T_{\max }\right)$

3.1

$r_{\min }$

1.6

$x_{\text {pre }}$

0.0001

${ }^{a} m$ is the sample mass; $V_{\mathrm{i}}$ is the internal volume of the calorimeter vessel; $T_{\text {cal }}$ is the temperature of the calorimeter when sealed; $p_{\text {cal }}$ is the pressure of the helium and sample when sealed; $r\left(T_{\max }\right)$ is the ratio of the heat capacity of the full calorimeter to that of the empty at the highest temperature $T_{\max } \approx 524 \mathrm{~K}$ of these measurements; $r_{\min }$ is the minimum value of $r$ observed in this study; and $x_{\text {pre }}$ is the mole-fraction impurity used for pre-melting corrections. 


\section{TABLE 2}

Melting-study summary for xanthone ${ }^{a}$

\begin{tabular}{rr}
\hline$F$ & $T / \mathrm{K}$ \\
\hline 0.2046 & $448.755_{0}$ \\
0.4047 & $448.774_{5}$ \\
0.5547 & $448.780_{1}$ \\
0.7048 & $448.783_{3}$ \\
0.8548 & $448.785_{0}$ \\
$T_{\text {tp }}=\left(448.79_{5} \pm 0.02\right) \mathrm{K}^{b}$ \\
\multicolumn{2}{c}{$x=0.9999$} \\
\hline
\end{tabular}

a $F$ is the fraction melted at observed temperature $T, T_{\mathrm{tp}}$ is the triple-point temperature, $x$ is the derived mole-fraction purity of the sample.

$b$ The uncertainty is the expanded uncertainty with 0.95 level of confidence. 
TABLE 3

Molar heat capacities $C_{\text {sat,m }}$ at vapor-saturation pressure measured with adiabatic calorimetry for xanthone $\left(R=8.3144621 \mathrm{~J} \cdot \mathrm{K}^{-1} \cdot \mathrm{mol}^{-1}\right)^{a}$

\begin{tabular}{|c|c|c|c|c|c|c|c|}
\hline$N^{b}$ & $<T>/ \mathrm{K}$ & $C_{\mathrm{sat}, \mathrm{m}} / R^{c}$ & $\Delta T / \mathrm{K}$ & $\mathrm{N}^{b}$ & $\langle T>/ \mathrm{K}$ & $C_{\mathrm{sat}, \mathrm{m}} / R^{c}$ & $\Delta T / \mathrm{K}$ \\
\hline \multicolumn{8}{|c|}{ crystal(II) } \\
\hline 10 & 4.948 & 0.050 & 1.102 & 2 & 107.097 & 10.257 & 9.739 \\
\hline 10 & 5.884 & 0.088 & 0.892 & 2 & 116.862 & 10.994 & 9.773 \\
\hline 10 & 6.774 & 0.136 & 1.018 & 2 & 126.790 & 11.749 & 10.021 \\
\hline 10 & 7.756 & 0.202 & 1.063 & 2 & 136.843 & 12.522 & 9.896 \\
\hline 10 & 8.886 & 0.300 & 1.120 & 2 & 146.729 & 13.295 & 9.866 \\
\hline 10 & 10.012 & 0.420 & 1.069 & 2 & 156.734 & 14.087 & 10.070 \\
\hline 10 & 11.155 & 0.554 & 1.168 & 2 & 166.814 & 14.902 & 10.015 \\
\hline 10 & 12.420 & 0.709 & 1.327 & 2 & 176.933 & 15.728 & 10.106 \\
\hline 10 & 13.824 & 0.911 & 1.480 & 2 & 187.026 & 16.571 & 10.078 \\
\hline 10 & 15.408 & 1.133 & 1.752 & 2 & 197.157 & 17.433 & 10.184 \\
\hline 10 & 17.209 & 1.398 & 1.857 & 2 & 207.332 & 18.309 & 10.167 \\
\hline 10 & 19.141 & 1.687 & 2.005 & 2 & 217.492 & 19.195 & 10.158 \\
\hline 10 & 21.272 & 1.999 & 2.251 & 2 & 227.644 & 20.098 & 10.154 \\
\hline 10 & 23.691 & 2.359 & 2.582 & 2 & 237.794 & 20.998 & 10.155 \\
\hline 10 & 26.445 & 2.758 & 2.922 & 2 & 247.000 & 21.829 & 10.185 \\
\hline 10 & 29.516 & 3.185 & 3.219 & 2 & 257.215 & 22.770 & 10.193 \\
\hline 10 & 32.987 & 3.649 & 3.686 & 2 & 267.433 & 23.722 & 10.200 \\
\hline 10 & 36.761 & 4.125 & 3.865 & 8 & 271.494 & 24.108 & 10.097 \\
\hline 10 & 40.808 & 4.592 & 4.231 & 2 & 277.658 & 24.697 & 10.212 \\
\hline 10 & 45.210 & 5.087 & 4.575 & 8 & 281.579 & 25.098 & 10.111 \\
\hline 10 & 49.987 & 5.579 & 4.980 & 9 & 285.783 & 25.491 & 8.203 \\
\hline 10 & 55.295 & 6.089 & 5.638 & 2 & 287.891 & 25.693 & 10.220 \\
\hline 10 & 61.275 & 6.630 & 6.320 & 8 & 287.931 & 25.716 & 2.998 \\
\hline 2 & 67.424 & 7.162 & 6.111 & 8 & 290.919 & 26.012 & 2.978 \\
\hline 2 & 74.047 & 7.704 & 7.121 & 8 & 293.888 & 26.303 & 2.960 \\
\hline 2 & 81.422 & 8.298 & 7.617 & 8 & 296.837 & 26.623 & 2.939 \\
\hline 2 & 89.275 & 8.903 & 8.081 & 8 & 299.768 & 26.967 & 2.921 \\
\hline 2 & 97.771 & 9.553 & 8.904 & 8 & 302.679 & 27.291 & 2.902 \\
\hline \multicolumn{8}{|c|}{ crystal(I) } \\
\hline 8 & 308.544 & 26.522 & 2.944 & 3 & 416.688 & 35.189 & 10.198 \\
\hline 9 & 314.516 & 27.030 & 8.309 & 12 & 419.613 & 35.413 & 16.583 \\
\hline 2 & 319.049 & 27.448 & 10.319 & 2 & 422.488 & 35.639 & 10.229 \\
\hline 2 & 329.404 & 28.250 & 10.373 & 6 & 426.255 & 35.934 & 10.285 \\
\hline 2 & 339.755 & 29.101 & 10.317 & 3 & 426.893 & 35.967 & 10.173 \\
\hline 2 & 350.053 & 29.937 & 10.266 & 4 & 428.370 & 36.089 & 10.048 \\
\hline 2 & 360.249 & 30.758 & 10.111 & 2 & 432.603 & 36.416 & 9.990 \\
\hline 2 & 370.496 & 31.596 & 10.397 & 12 & 434.538 & 36.592 & 13.201 \\
\hline 2 & 380.935 & 32.427 & 10.464 & 6 & 436.392 & 36.756 & 10.132 \\
\hline
\end{tabular}


a The relative expanded uncertainty for the heat capacities $U_{\mathrm{r}}\left(C_{\mathrm{sat}, \mathrm{m}}\right)=0.03$ at $T / \mathrm{K}=5$, 0.005 at $T / \mathrm{K}=10$, and 0.001 for temperatures $T / \mathrm{K} \geq 30$ with 0.95 level of confidence. The standard uncertainty in the mean temperatures $u(\langle T\rangle) / \mathrm{K}=0.01$.

$b \quad$ Adiabatic series number.

$c$ Average heat capacity for a temperature increment of $\Delta T$ with a mean temperature $\langle T\rangle$. 
TABLE 4

Measurements of molar energy increment $\Delta_{\mathrm{tot}} U_{\mathrm{m}}$ and derived results for xanthone

\begin{tabular}{|c|c|c|c|c|c|c|}
\hline \multirow{2}{*}{$\mathrm{N}^{a}$} & \multirow{2}{*}{$\mathrm{h}^{b}$} & \multirow{2}{*}{$\frac{T_{\mathrm{i}}}{\mathrm{K}}$} & \multirow{2}{*}{$\frac{T_{\mathrm{f}}}{\mathrm{K}}$} & \multirow{2}{*}{$\frac{T_{\text {trs }}}{\mathrm{K}}$} & \multirow{2}{*}{$\frac{\Delta_{\mathrm{tot}} H_{\mathrm{m}}{ }^{c}}{\mathrm{~kJ} \cdot \mathrm{mol}^{-1}}$} & \multirow{2}{*}{$\frac{\Delta_{\mathrm{trs}} H_{\mathrm{m}}{ }^{d}}{\mathrm{~kJ} \cdot \mathrm{mol}^{-1}}$} \\
\hline & & & & & & \\
\hline \multicolumn{7}{|c|}{ single-phase measurements in phase cr(II) } \\
\hline 8 & 1 & 205.300 & 266.467 & & 10.605 & 0.003 \\
\hline 11 & 1 & 61.168 & 119.955 & & 4.388 & -0.001 \\
\hline 11 & 1 & 119.967 & 203.716 & & 10.122 & 0.000 \\
\hline 11 & 1 & 203.667 & 290.036 & & 15.703 & 0.003 \\
\hline \multicolumn{7}{|c|}{ cr(II)-to-cr(I) phase transition } \\
\hline 9 & 1 & 289.885 & 310.371 & 304.50 & 4.532 & 0.000 \\
\hline 11 & 1 & 290.047 & 310.534 & & 4.533 & 0.000 \\
\hline & & & & & average & 0.000 \\
\hline \multicolumn{7}{|c|}{ single-phase measurements in phase $\operatorname{cr}(\mathrm{I})$} \\
\hline 7 & 1 & 330.388 & 396.576 & & 17.071 & 0.002 \\
\hline 7 & 1 & 396.537 & 431.805 & & 10.263 & 0.000 \\
\hline 11 & 1 & 310.986 & 392.846 & & 20.477 & 0.002 \\
\hline 11 & 1 & 392.858 & 440.913 & & 14.070 & 0.000 \\
\hline \multicolumn{7}{|c|}{ cr(I)-to-liquid phase transition } \\
\hline 4 & 2 & 443.285 & 452.097 & $448.79_{5}$ & 30.362 & 27.478 \\
\hline 7 & 6 & 441.931 & 452.195 & & 30.813 & 27.475 \\
\hline 12 & 2 & 441.196 & 451.925 & & 30.941 & 27.471 \\
\hline & & & & & average & 27.475 \\
\hline \multicolumn{7}{|c|}{ single-phase measurements in the liquid phase } \\
\hline 14 & 1 & 451.180 & 491.439 & --- & 14.577 & 0.000 \\
\hline
\end{tabular}

${ }^{a}$ Adiabatic series number.

$b$ Number of heating increments.

c $\Delta_{\mathrm{tot}} H_{\mathrm{m}}$ is the molar energy input from the initial temperature $T_{\mathrm{i}}$ to the final temperature $T_{\mathrm{f}}$. The relative expanded uncertainty for $\Delta_{\mathrm{tot}} H_{\mathrm{m}}$ is 0.001 with 0.95 level of confidence.

${ }^{d} \Delta_{\mathrm{trs}} H_{\mathrm{m}}$ is the net molar enthalpy of transition at the transition temperature $T_{\mathrm{trs}}$ or the excess enthalpy for single-phase measurements relative to the heat-capacity curve described in the text and defined in table 5. 


\section{TABLE 5}

Molar thermodynamic functions at vapor-saturation pressure for xanthone $\left(R=8.3144621 \mathrm{~J} \cdot \mathrm{K}^{-1} \cdot \mathrm{mol}^{-1}\right)^{a}$

\begin{tabular}{|c|c|c|c|c|c|c|c|}
\hline$T / \mathrm{K}$ & $C_{\mathrm{sat}, \mathrm{m}} / R$ & $\Delta_{0}^{T} S_{\mathrm{m}} / R$ & $\Delta_{0}^{T} H_{\mathrm{m}} / R T$ & $T / \mathrm{K}$ & $C_{\mathrm{sat}, \mathrm{m}} / R$ & $\Delta_{0}^{T} S_{\mathrm{m}} / R$ & $\Delta_{0}^{T} H_{\mathrm{m}} / R T$ \\
\hline \multicolumn{8}{|c|}{ crystal(II) } \\
\hline 5.00 & 0.054 & 0.018 & 0.014 & 140.00 & 12.768 & 13.045 & 6.882 \\
\hline 10.00 & 0.418 & 0.143 & 0.107 & 160.00 & 14.350 & 14.852 & 7.716 \\
\hline 20.00 & 1.813 & 0.843 & 0.598 & 180.00 & 15.983 & 16.636 & 8.543 \\
\hline 30.00 & 3.251 & 1.854 & 1.247 & 200.00 & 17.677 & 18.407 & 9.372 \\
\hline 40.00 & 4.502 & 2.967 & 1.909 & 220.00 & 19.418 & 20.173 & 10.205 \\
\hline 50.00 & 5.581 & 4.090 & 2.539 & 240.00 & 21.196 & 21.939 & 11.047 \\
\hline 60.00 & 6.517 & 5.192 & 3.125 & 260.00 & 23.028 & 23.708 & 11.898 \\
\hline 70.00 & 7.375 & 6.262 & 3.672 & 280.00 & 24.930 & 25.483 & 12.760 \\
\hline 80.00 & 8.185 & 7.300 & 4.186 & 298.15 & 26.772 & 27.105 & 13.556 \\
\hline 90.00 & 8.960 & 8.309 & 4.673 & 300.00 & 26.980 & 27.271 & 13.638 \\
\hline 100.00 & 9.722 & 9.292 & 5.140 & $304.50^{b}$ & 27.521 & 27.677 & 13.839 \\
\hline 120.00 & 11.232 & 11.198 & 6.029 & & & & \\
\hline \multicolumn{8}{|c|}{ crystal(I) } \\
\hline $304.50^{b}$ & 26.150 & 27.677 & 13.839 & 400.00 & 33.912 & 35.841 & 17.722 \\
\hline 320.00 & 27.516 & 29.009 & 14.469 & 420.00 & 35.441 & 37.532 & 18.529 \\
\hline 340.00 & 29.121 & 30.726 & 15.284 & 440.00 & 37.021 & 39.218 & 19.334 \\
\hline 360.00 & 30.740 & 32.436 & 16.097 & $448.79_{5}^{b}$ & 37.687 & 39.957 & 19.687 \\
\hline 380.00 & 32.354 & 34.142 & 16.911 & & & & \\
\hline \multicolumn{8}{|c|}{ liquid } \\
\hline $448.79_{5}^{b}$ & 42.422 & 47.320 & 27.050 & 500.00 & 44.990 & 52.039 & 28.756 \\
\hline 460.00 & 42.977 & 48.373 & 27.431 & 520.00 & 45.975 & 53.823 & 29.399 \\
\hline 480.00 & 43.988 & 50.223 & 28.100 & & & & \\
\hline
\end{tabular}

a Relative expanded uncertainties for all properties are 0.03 at $T=5 \mathrm{~K}, 0.005$ at $T=$ $10 \mathrm{~K}$, and 0.001 for temperatures $T \geq 30 \mathrm{~K}$ with 0.95 level of confidence.

b

Values at this temperature were calculated with heat capacities extrapolated linearly. 
TABLE 6

Calculated fundamental vibrational wavenumbers $\bar{v}$ used in the computation of the idealgas entropies for xanthone ${ }^{a}$

\begin{tabular}{rrrrl}
\hline $\bar{v} / \mathrm{cm}^{-1}$ & $\bar{v} / \mathrm{cm}^{-1}$ & $\bar{v} / \mathrm{cm}^{-1}$ & $\bar{v} / \mathrm{cm}^{-1}$ & $\bar{v} / \mathrm{cm}^{-1}$ \\
\hline 61.7 & 535.5 & 873.3 & 1208.4 & 1609.6 \\
116.0 & 585.0 & 920.7 & 1210.5 & 1620.7 \\
145.0 & 624.6 & 955.5 & 1234.2 & 1680.4 \\
225.6 & 647.1 & 957.3 & 1269.4 & 3111 \\
238.6 & 665.0 & 978.7 & 1317.8 & 3111 \\
281.4 & 693.4 & 979.6 & 1341.1 & 3124 \\
311.2 & 708.6 & 1023.0 & 1346.9 & 3124 \\
375.1 & 751.0 & 1027.1 & 1457.3 & 3135 \\
409.4 & 758.8 & 1096.7 & 1457.7 & 3135 \\
434.9 & 792.4 & 1105.4 & 1467.9 & 3139 \\
448.4 & 826.4 & 1143.3 & 1478.8 & 3139 \\
513.8 & 858.4 & 1146.9 & 1570.4 & \\
515.2 & 862.4 & 1170.6 & 1591.9 & \\
\end{tabular}

a The scale factor 0.975 was used in the calculation of the listed wavenumbers. The rotational constants are $A=0.04858 \mathrm{~cm}^{-1}, B=0.01543 \mathrm{~cm}^{-1}$, and $C=0.01171 \mathrm{~cm}^{-1}$, and the symmetry number for xanthone is 2 . 


\section{TABLE 7}

Comparison of literature enthalpies of sublimation and values derived in this research.

\begin{tabular}{|c|c|c|c|c|c|}
\hline$T / \mathrm{K}$ & $\Delta_{0}^{T} S_{\mathrm{m}}(\mathrm{cr}) / R^{a}$ & $S_{\mathrm{comp}} / R^{\mathrm{b}}$ & $\Delta_{0}^{T} S_{\mathrm{m}}^{\mathrm{o}}($ ideal $) / R^{c}$ & $\Delta_{\mathrm{cr}}^{\mathrm{g}} S_{\mathrm{m}} / \mathrm{R}^{d}$ & $\Delta_{\mathrm{cr}}^{\mathrm{g}} H_{\mathrm{m}} /\left(\mathrm{kJ} \cdot \mathrm{mol}^{-1}\right)$ \\
\hline \multirow[t]{3}{*}{352.2} & $(31.77 \pm 0.03)$ & $(-12.53 \pm 0.10)$ & $(54.11 \pm 0.11)$ & $(34.87 \pm 0.13)$ & $(102.1 \pm 0.4)^{e}$ \\
\hline & & & & Monte et al.[9] & $(103.9 \pm 0.6)$ \\
\hline & & & \multicolumn{2}{|c|}{ Sabbah and El Watik [7] } & $(97.1 \pm 0.3)$ \\
\hline \multirow[t]{3}{*}{298.15} & $(27.11 \pm 0.03)$ & $(-18.97 \pm 0.10)$ & $(49.93 \pm 0.10)$ & $(41.80 \pm 0.14)$ & $(103.6 \pm 0.4)^{e}$ \\
\hline & & & \multicolumn{2}{|c|}{ Freitas et al.(original) [8] } & $(102.7 \pm 2.3)^{f}$ \\
\hline & & & \multicolumn{2}{|c|}{ Freitas et al.(revised here) } & $(104.2 \pm 1.3)^{g}$ \\
\hline
\end{tabular}

a Standard entropy of crystalline xanthone interpolated from table 5.

$b$ Entropy of compression calculated with the sublimation pressures $p$ reported by Monte et al.[9]. $S_{\text {comp }}$ is $\ln \left(p / p^{\circ}\right)$, where $p^{\circ}$ is $101.325 \mathrm{kPa}$.

c Standard entropy of the ideal gas computed with parameters given in table $6\left(p^{\circ}=101.325 \mathrm{kPa}\right)$ and the methods described in the text.

$d$ Entropy of sublimation calculated with the values given in this table and equation 1.

$e \quad$ Enthalpy of sublimation $\Delta_{\mathrm{cr}}^{\mathrm{g}} H_{\mathrm{m}}$ calculated with $T \cdot \Delta_{\mathrm{cr}}^{\mathrm{g}} S_{\mathrm{m}}$.

$f$ Enthalpy of sublimation based on the calorimetric results and computations reported by Freitas [8]

$g$ Enthalpy of sublimation based on the calorimetric results by Freitas [8] and the computed gasphase enthalpies of the present research. 


\section{TABLE 8}

Comparison of enthalpies of formation based on measured enthalpies of combustion for xanthone reported in the literature ${ }^{a}$

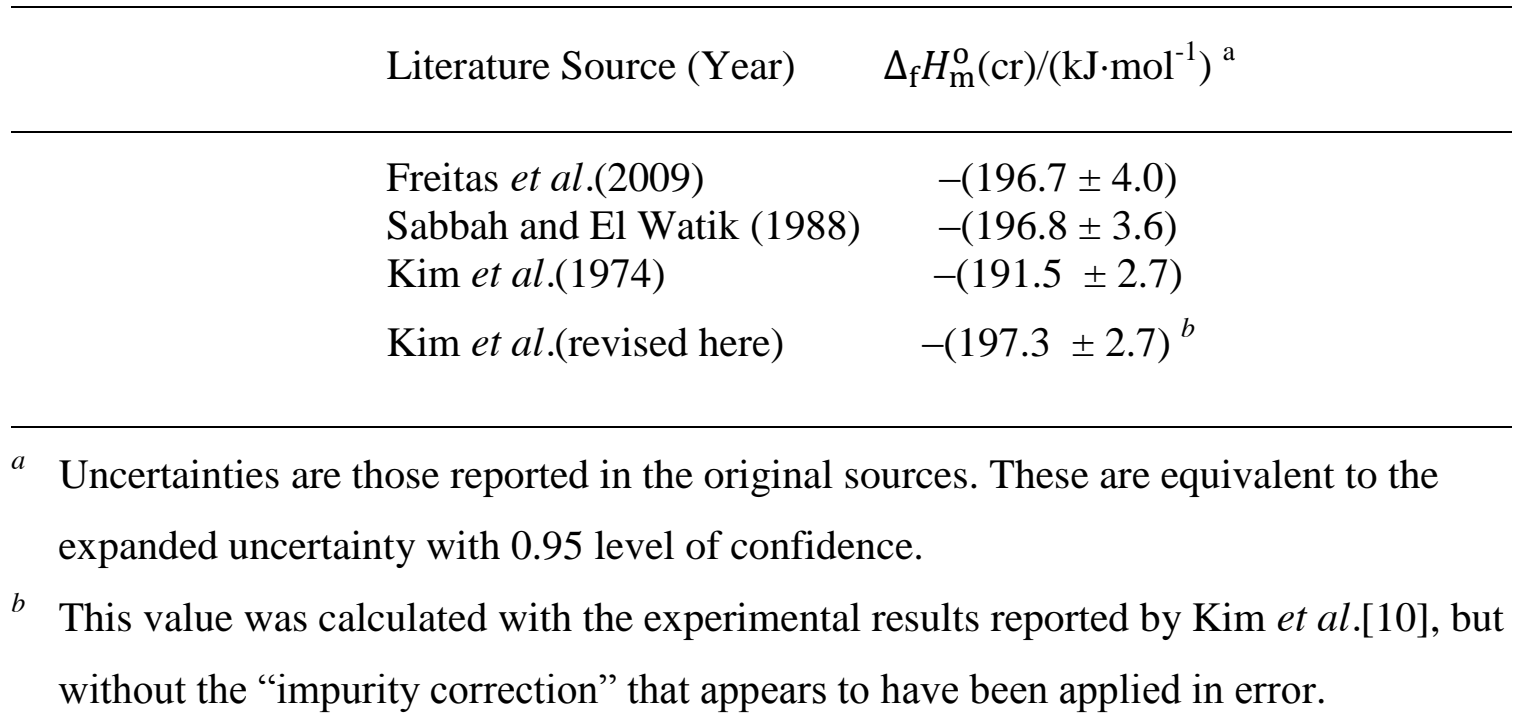




\section{Figure}

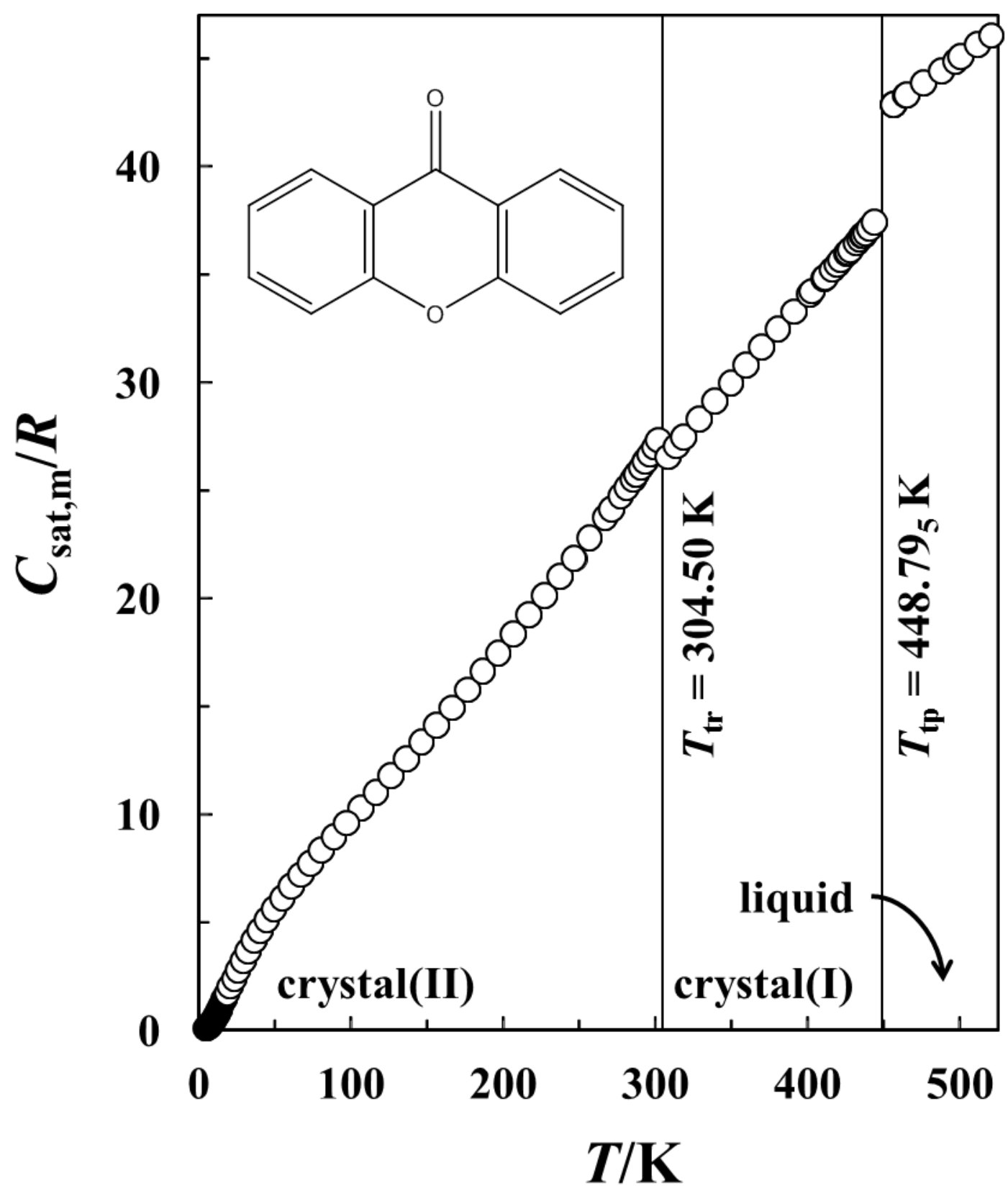

FIGURE 1. Plot of molar heat capacities against temperature at saturation pressure $C_{\mathrm{sat}, \mathrm{m}}$ for xanthone measured in this research. The vertical lines indicate the $\mathrm{cr}(\mathrm{II})$-to-cr(I) transition temperature $T_{\text {tr }}$ and triple-point temperature $T_{\mathrm{tp}}$. $\bigcirc$, Experimental values determined in this research. 